\title{
Research on the Changes of Serum Immunoglobulin Levels and Clinical Significance in Patients with Chronic Hepatitis B
}

\author{
Yanmei Guo, Yongwei Li, Yulin Yang, Yanqing Ma
}

Henan Province Hospital of Traditional Chinese Medicine, Zhengzhou, Henan, 450002

Keywords: serum immunoglobulin level; clinical significance; chronic hepatitis B

\begin{abstract}
Objective: To explore the significance of serum immunoglobulin test in the diagnosis and treatment of hepatitis B patients. Methods: Eighty patients with chronic hepatitis B were selected as study subjects. They were divided into study group A and study group B, with 40 cases in each group. The study group A patients were mild chronic hepatitis B; the study group B patients had severe chronic hepatitis B; 40 healthy people were also selected as the control group. Blood samples were collected from 3 groups of subjects and immunoglobulin (IgA, IgG, IgM) levels, total bilirubin (TBIL) levels, and prothrombin time (PTA) were measured. The test results of the three groups of subjects were compared with the level of immune function after symptomatic treatment. Results: The IgA, IgG, and IgM levels in study group A and study group B were higher than those in the control group, and the indexes in study group B were the highest; after treatment, the effective patients' IgA, IgG, IgM, and TBIL levels were lower than those in the invalid group. Patients, while PTA was higher than that of ineffective patients, the difference was statistically significant $(\mathrm{P}<0.05)$. Conclusion: To test the level of serum immunoprotein in patients with hepatitis $\mathrm{B}$ can provide scientific basis for the diagnosis of the disease and evaluation of the therapeutic effect, and has high clinical application value.
\end{abstract}

\section{Introduction}

Hepatitis B is a global disease caused by the hepatitis B virus and has a relatively high incidence in developing countries. The number of asymptomatic hepatitis B virus carriers in the world is $>$ 200 million, while in China $>100$ million, including About 30 million patients with hepatitis B have liver damage [1]. Hepatitis B will spread through the blood, mothers and infants, causing hepatitis inflammatory disease and involving other organs, and even transform into cirrhosis or liver cancer. It is a worldwide epidemic disease. As children and young adults are the main targets of hepatitis B virus, it is incurable and repetitive, with great harm. Therefore, it is extremely important for the prevention and early diagnosis and treatment of hepatitis B. Immunoglobulins have an antibody activity as a manifestation of antibodies, but synthesis can be significantly increased when infection occurs in the body. Therefore, the detection of immunoglobulins in patients with hepatitis B has a clear guiding role for patients and has been clinically used. For the detection of infectious diseases. Studies have also confirmed that the higher the severity of liver parenchymal destruction, the higher the degree of increase in immunoglobulins, and the obvious correlation between them, it is an important indicator of diagnosis and treatment of chronic hepatitis B. TBIL can be used clinically to diagnose liver diseases and biliary abnormalities. When total body bilirubin rises, the patient will experience jaundice. Hepatitis B patients are unable to completely convert indirect bilirubin due to liver cell damage, resulting in high total bilirubin. Therefore, TBIL is an important indicator for the diagnosis and treatment of patients with hepatitis B. PTA as a liver-dependent vitamin dependent factor can also effectively reflect the degree of liver damage and its function, especially for patients with chronic severe hepatitis B and cirrhosis, PTA levels will be significantly reduced, there are reports in the literature, liver cell necrosis The definite limit is PTA $<40 \%$ [2].

\section{Research Methods}

Twenty-two patients with hepatitis B from June 2011 to October 2011 were selected to conduct 
serum immunoglobulin level change experiments. Of the 52 patients with hepatitis $B, 32$ were male and 20 were female. The patient's age ranged from 24 to 68 and the average age was 42 years. The weight is between $49 \mathrm{~kg}$ and $84 \mathrm{~kg}$, and the average weight is $62 \mathrm{~kg}$. According to different conditions, the patients were divided into two groups. Group A was acute hepatitis B patients, and group B was chronic hepatitis B patients, including 18 patients in group A and 34 patients in group B. After experiencing 52 cases of hepatitis B patients, 9 cases of persistent low-grade fever, 11 cases of muscle and joint aches, 15 cases of hepatomegaly, and 10 cases of abdominal pain and nausea were found. In addition, 20 healthy blood donors were randomly selected as the control group (regular group), including 12 male blood donors and 8 female blood donors. There was no significant difference $(\mathrm{P}>0.05)$ between the three groups in terms of gender, age, and weight, and they were comparable [3].

The Olympus AU2700 automatic biochemical analyzer manufactured by Japan Olympus Corporation was used. The reagents were manufactured by Desai Diagnostic Systems (Shanghai) Co., Ltd.

After the patient got up in the morning, he took an empty stomach and used a vacuum blood collection tube without additives to remove $3 \mathrm{ml}$ of venous blood from the patient's body and seal it at room temperature. According to serum total bilirubin, transaminase and other biochemical indicators and HBsAg quantification combined with clinical symptoms, patients will be divided into two groups, A group is hepatitis B acute group, B group is chronic hepatitis B group, using serum immunoturbidimetric method The levels of serum immunoglobulin IgG, IgA, and IgM were measured. The level of serum immunoglobulin levels was changed to observe the degree of liver function intact and impaired in patients with hepatitis B.

Statistical data and SPSS 12.0 were used for statistical analysis. The data were expressed as mean \pm standard deviation $(\mathrm{x} \pm \mathrm{s})$. The count data were analyzed using the $\chi 2$ test. $\mathrm{P}<0.05$ indicated that the difference was statistically significant.

Randomly selected 58 patients with hepatitis B who were treated in our hospital from April 2014 to October 2015 as the observation group. All patients in this group were diagnosed as chronic hepatitis B by clinical examination. There were 31 male patients and 27 female patients. The patient's age ranged from 23 to 67 years and the average age was (43.8 \pm 5.4$)$ years. Body weight: 47 to $88 \mathrm{~kg}$, average body weight $(62.4 \pm 13.6) \mathrm{kg}$. At the same time, 58 healthy persons were used as the control group. There were no severe diseases such as liver, kidney, heart and lung in this group of patients, and no antimicrobial or immunomodulatory drugs were used in this study. There were 31 male patients and 27 female patients. The patient's age ranged from 21 to 66 years and the average age was $(43.7 \pm 4.5)$ years. Weight: 48 to $86 \mathrm{~kg}$, average weight $(62.3 \pm 13.9) \mathrm{kg}$. Patients were divided into an improved group $(n=37)$ and a non-impaired group $(n=21)$ based on the "Guidelines for the Prevention and Treatment of Chronic Hepatitis B (2010 edition)". The improvement group mainly included patients who recovered and had effective treatment, including 21 male patients and 16 female patients. The patient's age ranged from 21 to 68 years and the average age was $(43.5 \pm 4.6)$ years. Weight: 48 to $85 \mathrm{~kg}$, average body weight $(62.2 \pm 13.4) \mathrm{kg}$. In the non-impaired group, there were mainly patients with ineffective treatment and worsening of the disease, including 12 cases of male patients and 9 cases of female patients. The patient's age ranged from 22 to 69 years and the average age was $(43.9 \pm 4.8)$ years. Weight: 46 to $87 \mathrm{~kg}$, average body weight $(62.5 \pm 13.7) \mathrm{kg}$. There was no statistically significant difference between the groups $(\mathrm{P}>$ 0.05), and they were well comparable.

All patients were tested for serum immunoglobulin levels and $3 \mathrm{ml}$ of blood was collected on an empty stomach in the early morning. After centrifugation, biochemical analyzers and related reagents produced by Shanghai Kehua Biochemical Co., Ltd. were used to strictly perform IgG, IgA, and IgM levels according to standard operating procedures. Inspect and record relevant data. SPSS 19.0 software was used for processing. Count data was expressed as a percentage and $\chi 2$ test was used. The mean ( \pm standard deviation) is used as a measure of the measurement data and a t-test is used. $\mathrm{P}<0.05$ indicates a statistically significant difference. Hepatitis B is a disease caused by hepatitis B virus infection and resulting in damage to liver cells. It is usually divided into two 
groups: acute hepatitis B and chronic hepatitis B. Chronic hepatitis B is usually difficult to cure and prone to relapse. Symptoms, so in the clinical treatment of chronic hepatitis B is of great significance. Immunoglobulins are a class of antibody analogues with significant antibody activity. When an infection occurs in the body, a large number of immunoglobulins are synthesized. Therefore, the use of immunoglobulin for the detection of hepatitis B patients has a high clinical guiding value [4].

\section{Results}

A clinical trial of serum globulin levels was performed in groups A, B, and the control group. The results of the trial were analyzed and analyzed. Patients with acute hepatitis B (group A) and patients with chronic hepatitis B (group B) were found to have serum levels. The relevant values of immunoglobulins, such as IgM, IgA, IgG, etc., were significantly higher than those of healthy blood donors. There was a statistically significant difference $(\mathrm{P}<0.05)$. The level of immune function in patients with different therapeutic effects was analyzed after treatment. Patients had lower IgA, IgG, IgM, and TBIL than those who did not respond, and PTA was higher than ineffective patients. The difference was statistically significant $(\mathrm{P}<0.05)$.

Compared with acute hepatitis B, chronic hepatitis B is highly infectious, difficult to treat, and has a high recurrence rate. Therefore, it has become the focus of prevention and treatment in medical institutions [5]. Immunoglobulin is a tetrapeptide chain molecule, which is similar to an antibody and has an antibody activity. By combining with an antigen, it can exert an immune effect and mediate an immune response. In human serum, immunoglobulins mainly include IgA, IgG, and IgM. They have different characteristics and different divisions of labor. However, once the body is infected and invaded, the amount of synthesis of these proteins will increase significantly. For the time being, detecting immunoglobulin levels in patients has a clinical guiding role; and studies have confirmed that the more serious the liver function damage of patients, the more obvious these indicators increase. The results of this study show that the levels of IgA, IgG, and IgM in the study group A and study group B patients are higher than those in the control group, and the indexes of the study group B patients are the highest. PTA is involved in liver vitamin synthesis activities and can reflect the patient's liver function and degree of damage, especially in severe patients, cirrhosis patients, PTA significantly reduced. The results of this study also showed that the levels of IgA, IgG, IgM, and TBIL in patients with effective treatment were lower than those in patients who did not respond to treatment, and PTA was higher than in patients who did not respond to treatment. This is consistent with the literature. Hepatitis B is caused by the body's infection with hepatitis B virus. Hepatitis $\mathrm{B}$ virus has caused many special immune reactions to cause pathological immune damage in liver tissue. It is not caused by the direct damage to liver cells caused by hepatitis B virus. The main pathogenesis of the disease is immune dysfunction. Clinically relevant studies have found that the levels of immunoglobulins in patients with chronic hepatitis B are higher than those in healthy people. The main reason for this is the liver Kupffer cell dysfunction in patients with chronic hepatitis B. In this study, serum immunoglobulin levels were measured in both hepatitis B patients and healthy patients. The results showed that the levels of IgG, IgA, and IgM in the observation group were improved compared with the control group. In addition, the improvement of the condition improved the levels of IgG, IgA, and IgM in the patients and those who did not improve. The results showed that all the above indicators were reduced. From the above research results, it can be proved that the detection of serum immunoglobulin levels has a guiding role in the clinical diagnosis and treatment of hepatitis B.

\section{Conclusion}

The determination of immunoglobulins is of great importance to the clinical diagnosis and treatment of hepatitis B. It can be used to determine the condition of patients by detecting serum immunoglobulin levels, and provide the necessary basis for clinical treatment. 


\section{References}

[1] John T, Parissis MD. Clinical characteristics and predictors of in-hospital mortality in acute heart failure with preserved left ventricular rejection fraction [J]. Am J Cardiol, 2011, 107(1): 79-84.

[2] Hogg K, Swedberg K, Mcmurray J. Heart failure with preservedleft ventricular systolic function, epidemiology, clinical characteristics, and prognosis [J]. J Am Coll Cardiol, 2004, 43(3): 317-327.

[3] Tribouiiloy C, Rusinaru D, Mahjoub H, et al. Prognosis of heartfailure with preserved ejection fraction: a 5 years prospective population-based study [J]. Eur Heart J,2008,29(3): 339-347.

[4] Remme WJ, Swedberg K. Guidelines for the diagnosis and treatment of chronic heart failure[J]. Eur Heart J, 2001, 22 (17) :1527-1560.

[5] Elesher AA, Redtield MM, Rihal CS, et al. Coronary endothelialdys function and hyperlipidemia are independently associated withdiastolic dysfunction in humans [J]. Am Heart $\mathrm{J}$, 2007, 153(6) :1081-1087. 